\title{
Folding and crumpling of graphene under biaxial compression
}

\author{
J.A. Baimova, B. Liu, K. Zhou ${ }^{\dagger}$ \\ †kzhou@ntu.edu.sg
}

\begin{abstract}
Nanyang Technological University, Nanyang Avenue 50, 639798, Singapore, Singapore
This paper studies the effect of biaxial compression on the mechanical properties of three-dimensional structure consist of six graphene flakes with interlayer distance two times bigger than this of graphite. The folding and crumpling of graphene sheets under loading and unloading is studied. It is found that considered material cannot be elastically deformed even for low strain values. It is shown that wide ripples which appear at the first deformation stages transform to the sharp folds due to the van der Waals interaction between graphene sheets. The ridges and corners of folds have the highest potential energy and atoms with two nearest neighbors are concentrated in the edges of folds. Presented results can be applied to the investigations of crumpled grapheme properties, which is of high importance for energy storage, electronics and composites.
\end{abstract}

Keywords: crumpled graphene, mechanical properties, molecular dynamics.

\section{Introduction}

Since the synthesis of three-dimensional (3D) graphene nanostructures became available in the past few years, crumpled graphene and other $3 \mathrm{D}$ carbon nanostructures have attracted growing interest.

Crumpled graphene is a novel graphene-based material having unique properties which can be produced by different methods [1]. Regular sheet-like graphene materials can easily aggregate, making their surface area and processability sensitive to the processing history, while crumpled graphene is aggregation-resistant material and have a much more stable, consistently higher surface area and excellent solution processability that are less sensitive to processing history [2]. For example, crumpled graphene with sharp ridges, folds and vertices was studied in [3]. Crumpled graphene films have been already used in energy storage [4,5], electronics [6], and composites $[7,8]$. For instance, it was reported that crumpled graphene allows for the fabrication of polymer-graphene nanocomposite films with low $\mathrm{O}_{2}$ permeability and effective reduction of transparency [9]. Despite many interesting properties of crumpled graphene remain unknown so far.

It is well known that the physical properties of carbon nanostructures strongly depend on their geometrical structure, which can easily be changed by applying pressure or strain. As deformation of graphene can strongly affect its properties and the performance of graphene-based materials $[5,8,10,11-15]$, it is highly desirable to control reversible wrinkling and crumpling of graphene. It was shown that the rigid crumpled graphene balls are remarkably stable against mechanical deformation and ideal for energy storage and energy conversion. The degree of crumpling can strongly affect properties of graphene and the performance of graphene-based devices and materials [10, 4, 7]. Folding and crumpling are very similar in nature and the crumpling process can be viewed as arising from successive folding events. Graphene has the propensity for stable folding because of very small both bending rigidity and bending energy at folds, compensated by intersheet interaction via van der Waals forces $[16,17]$. Ripples, folds and scrolls were observed both in experimental and theoretical studies [18$25]$ and it was shown that they can significantly affect various properties of graphene [26-28].

From above mentioned it can be seen that studying of graphene crumpling and folding is very important and timely. Here we report an analysis of the mechanical properties of 3D graphene by means of molecular dynamics (MD) simulation. The pressure-density relations during both the loading and unloading processes are investigated.

\section{Simulation details}

The initial structure is obtained by stacking up the six graphene flakes, each of which consist of 3936 atoms, with the distance between flakes $h=7.85 \AA$ (see fig.1a). Chosen interlayer distance is two times bigger then this of graphite $\left(h_{\text {graphite }}=3.34 \AA\right)$. Considered initial interlayer distance is not typical for graphene structures and was chosen with the aim to construct the new 3D carbon polymorph with the novel interesting properties. Relaxation of this structure leads to the formation of pure graphite, but in this work such relaxation was not allowed. Moreover, this interlayer distance was the main factor for getting the final structure with given geometry. Periodic boundary conditions are applied to the computational cell along the $x, y$ and $z$ directions to avoid the free-surface effect. Density $\rho$ of the initial structure is equal to $1 \mathrm{~g} / \mathrm{cm}^{3}$.

In this work the large-scale atomic/molecular massively parallel simulator (LAMMPS) package [29] is used with the adaptive intermolecular reactive empirical bond order (AIREBO) potential [30]. The AIREBO potential has been shown to provide an accurate account of the chemical and mechanical behavior of hydrocarbons, including graphene and diamond [30-32]. AIREBO potential allows the nonbonded interactions to be turned smoothly on or off as bonding configurations change, moreover, considered potential has the term dependent on dihedral angles. In particular, AIREBO potential was developed to obtain a potential that could be used for simulations of reactions in condensed-phase hydrocarbon systems, including liquids, 
polymers, ets. Various phenomena, like thermal ripples, graphene morphology and thermal conductivity of hybrid graphene sheets has been successfully studied with this potential [33-35]. The Lennard-Jones potential is used to describe the van der Waals interactions between neighboring structural units with the parameter $r=3.4 \AA$, at with the minimal potential energy of $-0.024 \mathrm{eV}$. A time step of $0.2 \mathrm{fs}$ is used. The Nose-Hoover thermostat is used to control the system temperature around $300 \mathrm{~K}$.

The biaxial compression is loaded to the computational cells by applying strain $\varepsilon_{x x}=\varepsilon_{y y}=-\varepsilon$ and $\varepsilon_{z z}=0$ at the strain rate of $\dot{\varepsilon}=0.02 \mathrm{ps}^{-1}$. Thelongitudinal $\left.\left(\sigma_{L}=\left(\sigma_{x x}+\sigma_{y y}\right) / 2\right)\right)$ and transverse $\left(\sigma_{T}=\sigma_{z z}\right)$ components of the resulting normal stress are determined during compression and unloading.

\section{Simulation results}

Figure $1 \mathrm{~b}$ presents the longitudinal stress $\sigma_{L}$ as the function of density for unloading (dashed lines) in comparison with loading (solid lines). Considered $3 \mathrm{D}$ structure can be biaxially compressed till the density $\rho=3 \mathrm{~g} / \mathrm{cm}^{3}$. To study the elasticity limit, the unloading that starts from different levels of biaxial compression is modeled. In fig. $2 b$, the deviation of the unloading curve from the loading one can be seen because of the structural changes in the material at first deformation stages. Even at low densities this structure cannot be elastically deformed.

In fig. 2 the structural transformations of single flake (shown by dark color in fig.1a) is presented for the densities (in $\mathrm{g} / \mathrm{cm}^{3}$ ) (a) 1.1, (b,e) 1.7, (c,d) 2.7 and (f) 1.2 for loading (a-c) and unloading which starts from $\varepsilon=0.2$ (d-f). Graphene sample obtains rippled structure at the first deformation stages. It can be seen that with the increase of strain, edges and corners of folds become sharp. The appearance of the defects took place under unloading, while there are almost no defects during compression. After $20 \%$ of unloading the structure has final density 1.2 , which means that additional tensile strain should be applied to get the initial density $\rho=1 \mathrm{~g} / \mathrm{cm}^{3}$. Even after additional deformation there are small ripples in each graphene sheet.

The final structure at $\rho=3 \mathrm{~g} / \mathrm{cm}^{3}$ is shown in fig.3a. The sharp edges of graphene flakes are shown schematically by red straight lines to visualize the shape of the structural units (graphene sheets). To study the peculiarities of graphene folding, one of the graphene flakes (shown by blue color in fig.1a) is presented in fig.3b. It can be seen that after compression graphene flakes have sharp edges. It is also found that for single graphene flake under compression no sharp corners and folds are formed. To produce such edged structure, two or more graphene sheets are required, which means that van der Waals forces can lead to the formation of sharp folds. The initial interlayer distance also can be considered as the factor affecting the formation of the structure with the sharp corners. However, this effect will be studied in detail in our further works.

Peculiarities of structural transformations are considered based on coordination numbers of atoms. The number of atoms each of which has $k$ nearest neighbors within its radius of $1.45 \AA$ is denoted by $N_{\mathrm{k}}$ and the total number of atoms in the bulk material is denoted by $N$. The ratios of $N_{2} / N$ and $N_{3} / N$

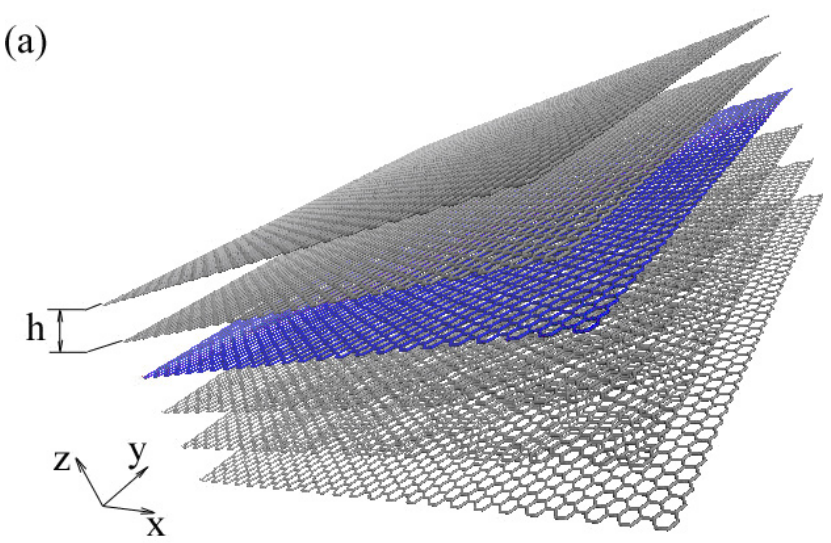

(b)

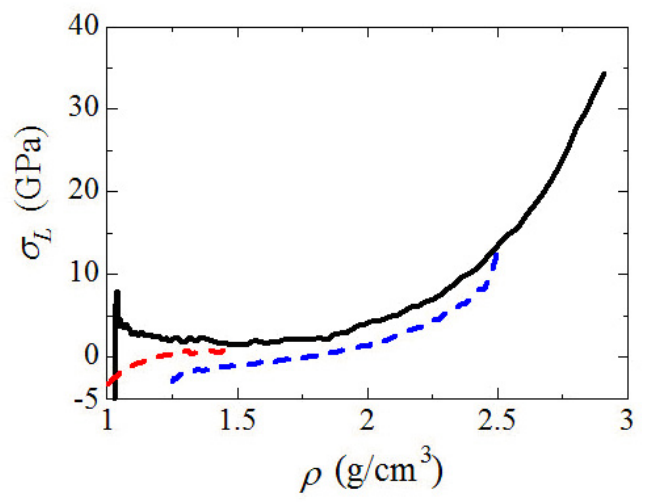

Fig. 1. (Color online) (a) Initial structure consist of six graphene flakes with the interlayer distance $h$. (b) The stress-density curves for loading (solid lines) and unloading (dashed lines).

as the functions of density is shown in fig.4a,b, respectively. It can be seen that both values are in good agreement with each other (with the increase of $N_{2} / N$ decrease of $N_{3} / N$ take place). It this case we do not consider atoms with $k=2$ as the atoms with broken bonds and $s p$-hybridization, but just like atoms with nearest neighbors within small radius. The additional analysis is showing that mostly, atoms with $k=2$ are placed on the top of the folds which are wide at low densities and sharp at final stages. This atoms still remain to be not near the defects of crystal structure, that is why no broken bonds can be seen in fig.2a-c. For low density, when there are small and wide ripples in the structure, the quantity of atoms with two nearest neighbors increase gradually until the density $2 \mathrm{~g} / \mathrm{cm}^{3}$ after which ripples tend to transform to the folds with sharp corners and most of carbon atoms rebuild their connections and $N_{2} / N$ decrease for $2<\rho<3 \mathrm{~g} / \mathrm{cm}^{3}$.

In fig. 5 the energy distribution during the compression is shown for densities (in $\mathrm{g} / \mathrm{cm}^{3}$ ) (a) 1.13, (b) 2, (c) 2.9. Energies $-7.8<E<-7.25,-7.25<E<-6.93,-6.9<E<-6.6$ and $E>-6.6 \mathrm{eV}$ are shown by green, red, black and blue colors, respectively. Ripples and edges of folds have the highest energies among the other atoms. Again, as for fig.4, atoms with $k=2$ are atoms with nearest neighbors within the radius $1.45 \AA$ without any broken bonds and no defects of crystal structure can be seen in fig.5. As it can be expected, the highest energies distributed on the corners and edges of the folds and the energy increase took place with the increase of strain. 


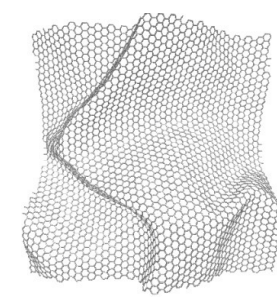

(a)

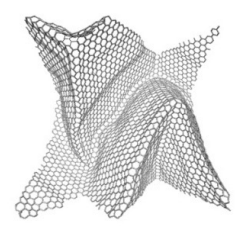

(d)

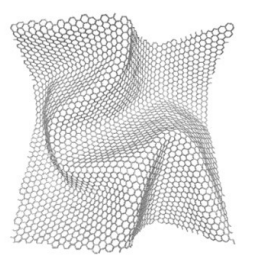

(b)

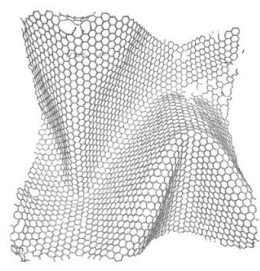

(e)

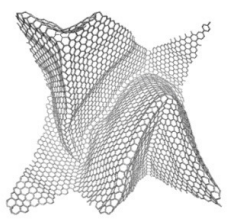

(c)

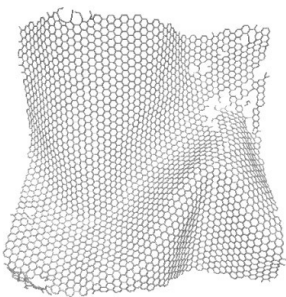

(f)
Fig. 2. (Color online) Structural transformations of the single graphene flake for the densities (in $\left.\mathrm{g} / \mathrm{cm}^{3}\right)(\mathrm{a}) 1.1$, (b,e) 1.7, (c,d) 2.7 and (f) 1.2 for loading (a-c) and unloading from strain $\varepsilon=0.2$ (d-f).
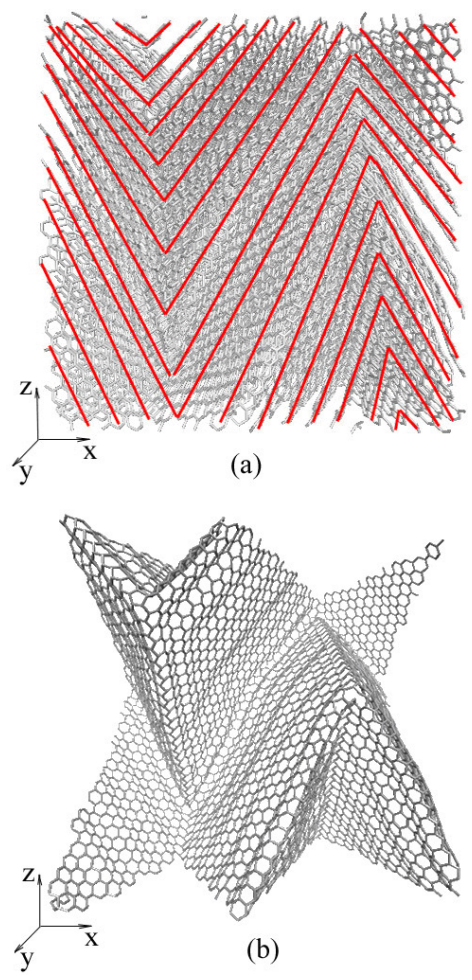

Fig. 3. (Color online) (a) Final structure at $\rho=2.9 \mathrm{~g} / \mathrm{cm}^{3}$. The sharp edges of graphene flakes are schematically shown by red straight lines. (b) The single graphene flake (shown by blue color in fig.1a).

\section{Discussion}

Methods of production of the novel 3D structures with given properties are of great interest nowadays as it can be seen form the multitude of works, both experimental and theoretical, aimed to this subject. The wide classification of carbon structures was given in [36,37], which can be considered as the guidance for the construction of new polymorphs. For example, some attempts to describe the mechanical properties of various $3 \mathrm{D}$ carbon nanostructures

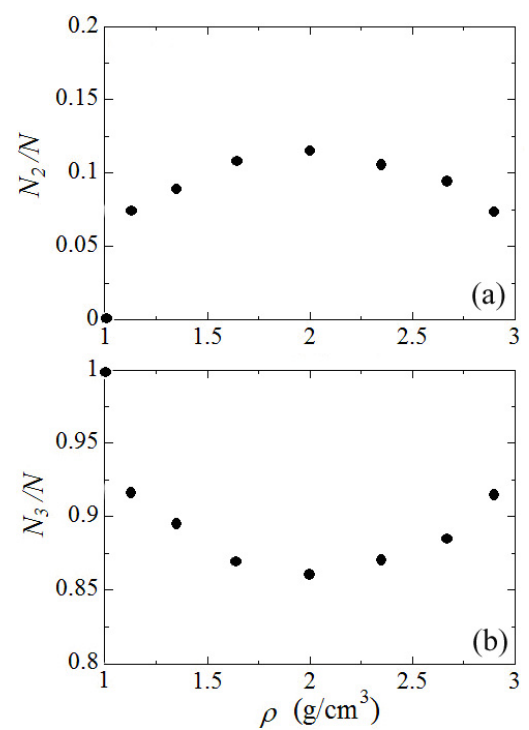

Fig. 4. Ratios of (a) $N_{2} / N$ and (b) $N_{3} / N$ as functions of the density.

based on carbon nanotubes, fullerenes and graphene flakes were made by authors in [38]. The structure investigated in present work can be considered both as the continuation of this attempts and efforts towards the studying of the ripples, wrinkles and folds.

The most studied 3D graphene structure is the crumpled graphene [40-41]. It was shown that the increasing stiffness and strength under compression is a unique feature of crumpled paper balls, which is exactly the same for our crumpled material.

On the other hand, ripples and folds, which are of great interest, was studied under different conditions, like substrates, elastic strain, etc., but many interesting properties are still remain unknown. In this work, one of the main factors affecting the process of folding was identified as interlayer distance which calls for further investigations.

\section{Conclusions}

In conclusion, $\mathrm{MD}$ simulation is performed to describe the peculiarities of folding and crumpling of graphene sheets in 3D material under compression. Mechanical properties and the peculiarities of structural transformations are studied during biaxial loading and unloading.

Stress-strain curves show that considered structure cannot be elastically strained even for low strain and the additional strain should be applied to get the plane graphene sheets after loading. The appearance of defects took place under unloading while under compression the structure of each graphene sheet remains defect-free.

After high strain the formation of folds with sharp edges and highest energies is shown. In comparison with the bulk structure consists of six graphene sheets it is shown that sharp folds cannot be observed for single graphene sheet under the same loading conditions. This can be explained by the van der Waals forces between graphene flakes in the 3D structure leading to the strong folding. 
(a)

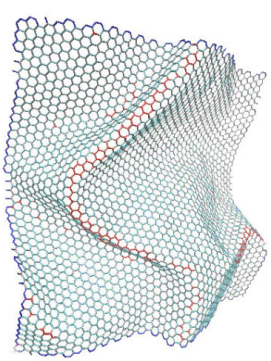

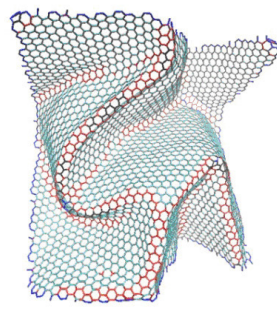

(b)

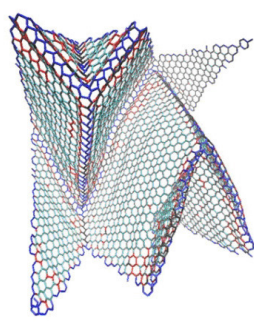

(c)
Fig. 5. (Color online) Potential energy of the atoms for densities (in $\mathrm{g} / \mathrm{cm}^{3}$ ) (a) 1.1, (b) 2, (c) 2.9. Energies $-7.8<E<-7.25,-7.25<E<-6.93$, $-6.9<E<-6.6$ and $E>-6.6 \mathrm{eV}$ are shown by green, red, black and blue colors, respectively.

Present study opens many new directions for the studying of the properties of rippled and wrinkled graphene flakes which are the main building units for crumpled graphene.

The authors gratefully acknowledge grant from Russian Scientific Fond №14-13-00982. All the calculations were carried out on the Supercomputer of the Supercomputer Center of Russian Academy of Science

\section{References}

1. J. Yan, Y. Xiao, G. Ning, T. Wei and Z. Fan. RSC Advances. 3, 2566 (2013).

2. L. Xiao, J. Damien, J. Luo, H. D. Jang, J. Huang, Z. He. Journal of Power Sources. 208, 187 (2012)

3. S. Cranford, M. Buehler. Phys. Rev. B. 84, 205451 (2011).

4. Y. Zhu, et al. Science. 329, 1537 (2011).

5. J. Luo, et al. ACS Nano. 5, 8943 (2011).

6. J. Miller, R. Outlaw, B. Holloway. Science. 329, 1637 (2010). 7. S. Stankovich, et al. Nature. 442, 282 (2006).

8. T. Ramanathan, et al. Nature Nanotech. 3, 327 (2008).

9. O.C. Compton, S. Kim, C. Pierre, J. M. Torkelson, S. T. Nguyen. Adv. Mater. 22, 4759 (2010).

10. J. Zang, S. Ryu, N. Pugno, Q. Wang, Q. Tu, M. J. Buehler, X. Zhao. Nat. Mater. 12, 321 (2013).

11. V. Rosato, M. Celino, G. Benedek, S. Gaito. Phys. Rev. B. 60, 16928 (1999).

12. A. Lundin, B. Sundqvist. Phys. Rev. B. 53, 8329 (1996).

13. O. F. Yagafarov, E. L. Gromnitskaya, A. G. Lyapin, et al. J. of Phys.: Conference Series. 215, 012054 (2010).

14. A. Ansari, B. Motevalli, A. Montazeri, S. Ajori. Solid State Commun. 151, 1141 (2011).
15. H. Sasakia, T. Hagia, K. Shintani. MRS Proceedings. (2010) mrsf12-1505-w10-27 (2013).

16. C. Liu, Z. Yu, D. Neff, A. Zhamu, B. Z. Jang. Nano Letters. 10, 4863 (2010).

17. X. Zhang, B. Wang, J. Sunarso,et al. Wiley Interdisciplinary Reviews: Energy and Environment. 1(3), 317 (2012).

18. A. Fasolino, J. Los, M. Katsnelson. Nat. Mater. 6, 858 (2007)

19. W. Bao, F. Miao, Z. Chen, H. Zhang, et al. Nature Nanotechnology. 4, 562 (2009).

20. J. Baimova, S. Dmitriev, K. Zhou. Phys. Status Solidi B. 249 (7), 1393 (2012).

21. F. Scarpa, S. Adhikari, A. J. Gil, C. Remillat. Nanotechnology. 21, 125702 (2010).

22. K. Kim, Z. Lee, B. Malone, K. Chan, B. Aleman, et al. Phys. Rev. B. 83, 245433 (2011).

23. J. Meyer, A. Geim, M. Katsnelson, K. Novoselov, T. Booth, S. Roth. Nature. 446, 60 (2007).

24. R. Kaner, L. Viculis, J. Mack. Science. 299, 1361 (2003).

25. X. Shi, N. Pugno, H. Gao. Journal of Computational and Theoretical Nanoscience. 7, 1 (2010).

26. M. Zwierzycki. J. Phys. Condens. Matter. 26 (13), 135303 (2014).

27. G. Gui, J. Zhong, Z. Ma. J. of Physics: Conference Series. 402, 012004 (2012).

28. P. Partovi-Azar, N. Nafari, M. Reza Rahimi Tabar. Phys. Rev. B. 83, 165434 (2011).

29. S. Plimpton. J. Comput. Phys. 117, 1 (1995).

30. S. Stuart, A. Tutein, et al. J. Chem. Phys. 112, 6472 (2000).

31. Z. Xu, M. Buehler. Nanotechnology. 20, 185701 (2009).

32. C.D. Reddy, A. Ramasubramaniam, V.B. Shenoy, Y. W. Zhang, Appl. Phys. Lett. 94, 101904 (2009).

33. A. K. Singh, R. G. Hennig. Phys. Rev. B. 87, 094112 (2013).

34. H. Wang, M. Upmanyu. Phys. Rev. B. 86, 205411 (2012).

35. B. Liu, J. A. Baimova, S. V. Dmitriev, X. Wang, H. Zhu, K. Zhou. J. Phys. D-Appl. Phys. 46 (30), 305302 (2013).

36. E. A. Belenkov, V.A. Greshnyakov. Phys. Solid State. 55 (8), 1754 (2013).

37. E. A. Belenkov, I. V. Shakhova. Phys. Solid State. 53 (11), 2385 (2011).

38. J.A. Baimova, B. Liu, S. V. Dmitriev, K. Zhou. pss (RRL). 8, 336 (2014).

39. A. Lobkovsky, S. Gentges, H. Li, D. Morse, T. A. Witten. Science. 270, 1482 (1995).

40. T. Tallinen, J. A. Astrom, et al. Nat. Mater. 8, 25 (2009).

41 K. Matan, R.B. Williams, T.A. Witten, S.R. Nagel. Phys. Rev. Lett. 88, 076101 (2002). 